\title{
The importance of plant genotype and contemporary evolution for terrestrial ecosystem processes
}

\author{
Connor R. Fitzpatrick, ${ }^{1,6}$ Anurag A. Agrawal, ${ }^{2}$ Nathan Basiliko, ${ }^{3}$ Amy P. Hastings, ${ }^{2}$ Marney E. Isaac, ${ }^{4}$ \\ Michael Preston, ${ }^{5}$ and Marc T. J. Johnson ${ }^{1}$ \\ ${ }^{1}$ Department of Biology, University of Toronto, Mississauga, Ontario L5L 1C6 Canada \\ ${ }^{2}$ Department of Ecology and Evolutionary Biology, Cornell University, Ithaca, New York 14853 USA \\ ${ }^{3}$ Department of Biology, Laurentian University, Sudbury, Ontario P3E 2 C6 Canada \\ ${ }^{4}$ Department of Physical and Environmental Sciences, University of Toronto-Scarborough, Toronto, Ontario M1C 1A4 Canada \\ ${ }^{5}$ Department of Geography, University of Toronto, Mississauga, Ontario L5L 1C6 Canada
}

\begin{abstract}
Plant genetic variation and evolutionary dynamics are predicted to impact ecosystem processes but these effects are poorly understood. Here we test the hypothesis that plant genotype and contemporary evolution influence the flux of energy and nutrients through soil, which then feedback to affect seedling performance in subsequent generations. We conducted a multiyear field evolution experiment using the native biennial plant Oenothera biennis. This experiment was coupled with experimental assays to address our hypothesis and quantify the relative importance of evolutionary and ecological factors on multiple ecosystem processes. Plant genotype, contemporary evolution, spatial variation, and herbivory affected ecosystem processes (e.g., leaf decay, soil respiration, seedling performance, $\mathrm{N}$ cycling), but their relative importance varied between specific ecosystem variables. Insect herbivory and evolution also contributed to a feedback that affected seedling biomass of $O$. biennis in the next generation. Our results show that heritable variation among plant genotypes can be an important factor affecting local ecosystem processes, and while effects of contemporary evolution were detectable and sometimes strong, they were often contingent on other ecological factors.
\end{abstract}

Key words: aboveground-belowground interactions; eco-evo dynamics; ecosystem genetics; evening primrose; extended phenotype; litter decay; soil ecosystem.

\section{INTRODUCTION}

Terrestrial ecosystems are shaped by complex interactions between biotic and abiotic factors operating above and belowground (Bardgett and Wardle 2010). As the basal resource in terrestrial food webs, plants provide the primary energy source for all communities, and in turn drive ecosystem processes involved in energy and nutrient cycling (Bardgett and Wardle 2010). Biologists have recognized intraspecific genetic variation in plants as a potentially important factor affecting primary productivity, nutrient cycling, and litter decay (Schweitzer et al. 2004, Crutsinger et al. 2006, Madritch et al. 2006). Based on these results, evolution within plant populations is predicted to have ecosystem-level consequences (Fussmann et al. 2007, Bailey et al. 2009). However, the importance of heritable plant traits and evolution for ecosystems, relative to the biotic and abiotic factors typically cited as most influential, is largely unknown (Hersch-Green et al. 2011, Matthews et al. 2011, Crutsinger et al. 2013).

Manuscript received 8 December 2014; revised 24 March 2015; accepted 31 March 2015. Corresponding Editor: P. B. Adler.

${ }_{6}^{6}$ E-mail: connor.fitzpatrick@mail.utoronto.ca
Intraspecific variation in plant growth and defense traits is expected to impact ecosystem processes because such variation can have direct consequences for soil nutrient input and the composition of decomposer communities, ultimately affecting litter decomposition and nutrient availability (Schweitzer et al. 2004). Natural selection that causes evolution of ecologically relevant plant traits (e.g., tissue chemistry and phenology) can potentially alter ecosystem function. For example, changes in plant secondary compounds can inhibit some groups of soil microbes while promoting the growth of others (Fierer et al. 2001). The effects may be particularly relevant because secondary metabolites exhibit consistently high heritability and thus are likely to rapidly evolve in response to selection (Geber and Griffen 2003). Although less studied, predicted ecosystem effects of phenological change are based on how phenology affects the timing of nutrient uptake and deposition in soils (Whitham et al. 2006). Ecosystemlevel effects of plant evolution could feedback to alter plant fitness if the traits subject to natural selection induce changes in the ecosystem, which in turn influence the fitness of focal individuals (i.e., an "eco-evolutionary feedback," sensu Fussman et al. [2007]). Although recent studies have found ecosystem effects of phenotypically diverged populations (Harmon et al. 2009, Post 
and Palkovacs 2009, Bassar et al. 2010), we are not aware of any study that has examined the effect of contemporary evolution on natural ecosystem processes and their potential feedback.

To understand the relevance of genotypic variation and evolution in plant traits for ecosystems, it is essential to quantify their importance relative to other ecological factors in natural environments (HerschGreen et al. 2011, Tack et al. 2011, Crutsinger et al. 2013). For example, herbivores can directly influence nutrient inputs into soil ecosystems through excretion, and indirectly via the effects of herbivory on the quantity and quality of plant litter (Hunter 2001, Yang and Gratton 2014). Similarly, ecosystem processes frequently vary in space due to variation in abiotic and biotic factors across the environment (Schlesinger et al. 1996, Vaieretti et al. 2013). Measuring the relative importance and interactions between genotypic variation, evolution and other ecological factors will provide a more complete understanding of terrestrial ecology and ecosystem functioning.

In this study, we sought to understand whether there is a dynamic feedback between evolution and ecosystem processes using common evening primrose (Oenothera biennis) as our focal plant species. We established 16 field plots, one-half of which had a five-year history of insect suppression (Agrawal et al. 2012). The herbivory treatment caused $O$. biennis populations to diverge in genotypic composition as well as chemical defense and phenological traits. Natural selection on $O$. biennis populations was caused directly by insect herbivores but also indirectly via changes in abundance of competing plant species. We tested the hypothesis that (1) plant genotypic variation in litter input and (2) the influence of contemporary evolution in plant populations affect ecosystem processes. To understand the importance of these effects, we compared the variation in ecosystem processes explained by plant genotype and evolution to the ecological effects of herbivore suppression and spatial variation in a field experiment. In characterizing ecosystem processes, we focused on components of energy flow (i.e., leaf decay, soil microbial activity) and nutrient cycling (i.e., $\mathrm{N}$ mineralization rate). We then tested if the soil ecosystem effects of evolution, insect herbivory, and spatial variation affect seedling performance in the next generation.

\section{Methods \\ Study system}

We used common evening primrose, Oenothera biennis L. (Onagraceae), as our focal species. Oenothera biennis is a native forb found throughout eastern North America in disturbed sites and old fields, where it forms discrete populations varying in size from one to several thousand individuals (Johnson 2011). Plants form a leafy rosette and produce a flowering stalk in their first or second year of growth, and then die following reproduction. Oenothera biennis exhibits a genetic system called permanent translocation heterozygosity, which renders individuals functionally asexual in that all seeds are genetically identical to one another (Cleland 1972). This means genetically identical individuals (hereafter referred to as genotypes) can be replicated from seed and evolution can be studied as the change in genotype frequency across generations. Within a population, genotypes of $O$. biennis show heritable variation for numerous phenotypic traits including flowering date, foliar $\mathrm{C}: \mathrm{N}$, biomass production, and a range of phenolic compounds (Johnson et al. 2009a).

\section{Experimental design}

The work presented here builds on a long-term field experiment first reported in Agrawal et al. (2012). The site was a fallow field located just outside Ithaca, New York, USA. The vegetation and land-use history were initially homogenous across the site. In May 2007, a total of 16 replicate $13.5-\mathrm{m}^{2}$ plots were plowed and planted with $O$. biennis. We started each plot with the identical composition of 18 genotypes in a central $1-\mathrm{m}^{2}$ area (3 individuals $\times 16$ genotypes +6 individuals $\times 2$ genotypes $=60$ individuals total per plot). The plots were surrounded with fencing to exclude deer and one-half of the plots were sprayed biweekly (April-October) with an insecticide (Asana XL; DuPont, Wilmington, Delaware, USA; final concentration $=0.00326 \%$ esfenvalerate $\mathrm{v} / \mathrm{v}$ with $\mathrm{H}_{2} \mathrm{O}$ ), to suppress insect herbivores. The insecticide was sprayed on aboveground foliar tissue and it rapidly degrades under UV light; its influence on the soil ecosystem was minimal as confirmed through a separate experiment (see Appendix A). Plants naturally reproduced and recruited via passive seed dispersal within plots each year, such that there was no manipulation to the plots after the experiment was started, other than the insecticide treatment. Colonization by other plant species occurred at the same time.

There was minimal dispersal between plots because $O$. biennis has no specialized dispersal mechanisms and the distance between plots was large. The abundance of $O$. biennis and the frequency of individual genotypes were quantified annually using microsatellite genotyping, which revealed three main patterns: (1) all populations evolved rapidly; (2) populations with and without insect herbivores genetically and phenotypically diverged from one another, such that populations experiencing ambient herbivory evolved increased phenolics and later flowering; and (3) decreased herbivory led to a substantial reduction in $O$. biennis accompanied by an increase in dandelion (Taraxacum officinale (Asteraceae)) abundance. The study presented here represents a novel extension to Agrawal et al. (2012), where all of the ecosystem assays and analyses were newly performed as described below.

In the summer of 2012, five years after the initiation of the experiment, we used field and laboratory assays to characterize ecosystem processes in the plots. In each 

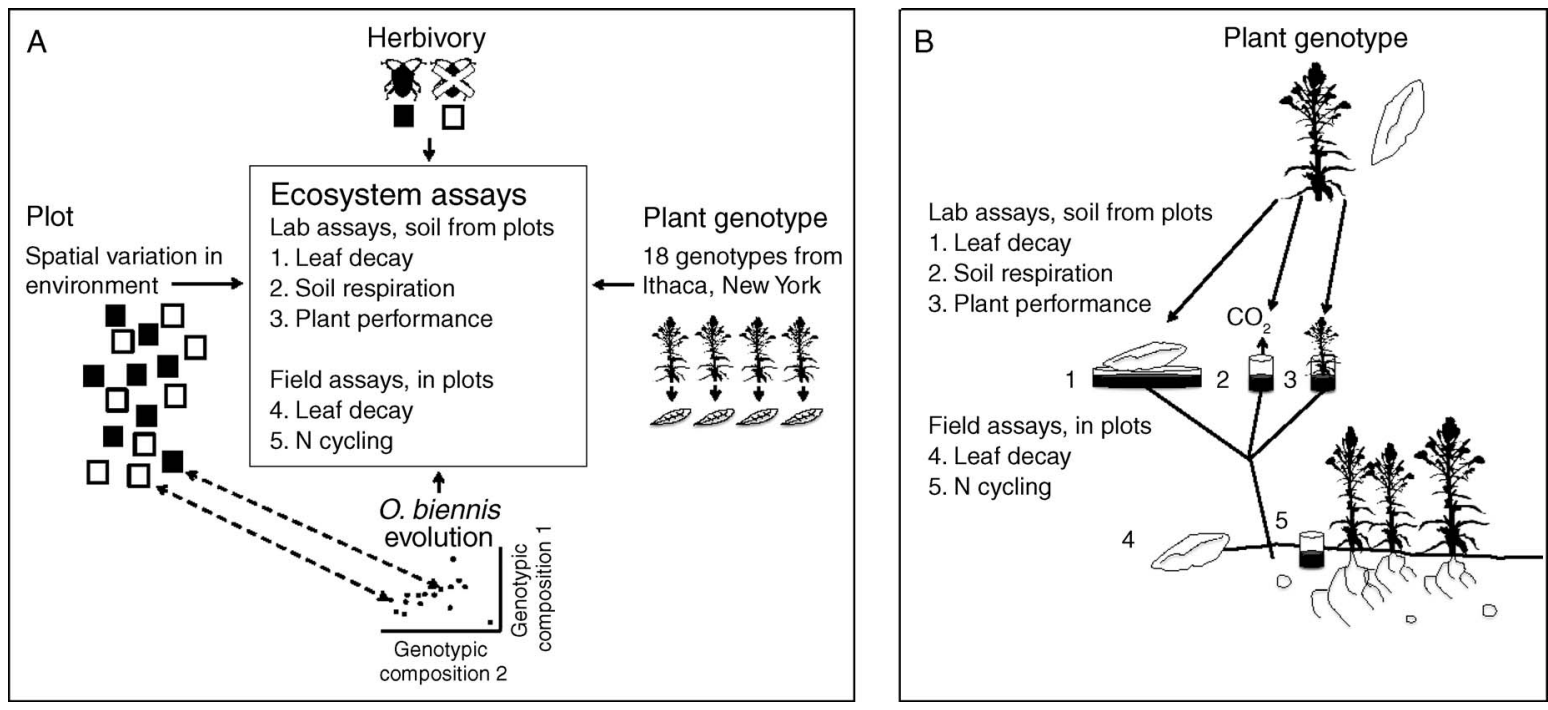

FIG. 1. A diagram representing the experimental design and ecosystem assay methods. (A) We studied the effects of four factors: plant genotype, contemporary evolution, herbivory, and spatial variation among plots. Herbivory represents the effect of insect suppression. Plant genotype is the effect of differences in tissue quality across Oenothera biennis genotypes. Plot is the effect of spatial variation in soil resources and conditions across the 16 plots. Finally, evolution is the effect of the unique $O$. biennis genotypic composition in each plot or the rate of evolutionary change within plots. Ecosystem effects of herbivory, plot, and evolution represent indirect effects mediated by changes to the soil ecosystem, while the effect of plant genotype is mediated by genotypic differences in tissue quality. (B) We used lab (1-3) and field-based (4 and 5) assays to test the effects of these factors on four distinct ecosystem processes.

assay, we assessed how four factors affected ecosystem processes (Fig. 1). These factors included (1) herbivore treatment (i.e., plots treated with insecticide or a water control); (2) plant genotype (i.e., O. biennis genotype leaf tissue used in assays); (3) plot (i.e., spatial location within the field); and (4) evolution measured as genotypic composition in 2011 and as Euclidean distance in genotype frequencies between the final and initial composition of each population (for details, see Appendix B: Table B1). We assessed genotypic composition by collecting tissue from 190 individual plants per plot and genotyping each sample using four microsatellite markers that provided a diagnostic identification of each of the 18 original genotypes (Agrawal et al. 2012). We then used correspondence analysis (CA) to quantify the genotypic composition (GC) of each plot based on the relative abundance of individual genotypes (Canoco for Windows version 4.55; Wageningen UR, Wageningen, Netherlands). CA is a multivariate ordination method that we used to depict the GC of each plot according to its score along the first two CA axes (Agrawal et al. 2012: Fig. 3A). Together these axes explain $56 \%$ of the variance in genotypic composition across plots. All plots were identical at the beginning of the experiment in 2007, so the position of plots along these axes in 2011 quantifies the degree of evolutionary divergence after four generations of evolution. These differences in genetic composition represented by the CA axes reflect the effects of selection by herbivores, competitors and spatial variation in the physical environment, as well as stochastic processes (i.e., genetic drift). As an additional metric of evolutionary change, we used Euclidean distance of each plot measured as the distance in genotypic composition between 2007 and 2011. Euclidean distance captures the magnitude and rate of change of each population irrespective of direction. Evolution measured as Euclidean distance was not significantly correlated with either axis of genotypic composition (GC1, $R^{2}=0.04, P=0.47$; GC2, $\left.R^{2}=0.22, P=0.07\right)$.

\section{Plant tissue and soil collections}

We used seeds from the collection of 18 genotypes used in Agrawal et al. (2012) for all experiments. These genotypes were initially collected from a $20 \mathrm{~km}$ radius surrounding Ithaca, New York (see Appendix A). In summer 2012, we germinated seeds on moistened filter paper in petri dishes exposed to natural sunlight. We then planted 10 seedlings per genotype individually into $15 \mathrm{~cm}$ diameter round pots $(500 \mathrm{~mL})$ filled with potting mix (Pro-Mix BX, Rivière-du-Loup, Quebec, Canada), and $0.2 \mathrm{~g}$ of slow-release fertilizer pellets (Miracle Gro14-14-14; Miracle Gro, Marysville, Ohio, USA). We grew plants on a rooftop at the University of Toronto Mississauga campus where they experienced ambient environmental conditions. We collected senesced leaf tissue from each plant over the course of the growing season and pooled tissue from the 10 individuals per genotype. All tissue was dried at $55^{\circ} \mathrm{C}$ for $72 \mathrm{~h}$ before weighing.

We collected soil used in our ecosystem assays from the experimental plots between insecticide spray treat- 
ments in early August 2012. We divided each plot into four quadrants and collected soil from a random location in each quadrant using a stainless steel soil corer $(5 \mathrm{~cm}$ radius $\times 10 \mathrm{~cm}$ length; Regular Soil Auger; AMS, American Falls, Idaho, USA). The corer was sterilized with $95 \%$ ethanol between plots. We stored the soil in Whirl Packs (sterile, polyethylene bags; Nasco, Fort Atkinson, Wisconsin, USA) on ice for $24 \mathrm{~h}$ before bringing it back to the lab. We then immediately sieved the soil to $2 \mathrm{~mm}$ and homogenized the samples to create a composite sample per plot, which was later used for all experimental assays. From this composite sample, we measured gravimetric soil moisture content ( $1 \mathrm{~g}$ of fieldmoist soil weighed before and after drying at $105^{\circ} \mathrm{C}$ for $24 \mathrm{~h}$ ), and $\mathrm{pH}$ ( $1 \mathrm{~g}$ field-moist soil in $10 \mathrm{~mL}$ of distilled $\mathrm{H}_{2} \mathrm{O}$ ) in triplicate for each plot. A subsample of soil from each composite plot sample was air dried and ground to a fine powder for elemental analysis. We used an elemental analyzer (ECS 4010 CHNSO Analyzer; Costech, Valencia, California, USA), to determine the total carbon and nitrogen content in the soil from each plot (Appendix B: Table B1).

\section{Leaf decay}

We assessed leaf decay in the field and in a laboratory setting (Fig. 1). In the field (Appendix B: Fig B1A), we created litter bags from window-screen mesh (mesh hole size $1.5 \mathrm{~mm}$ ). Each bag had three compartments adjacent to one another so that each compartment could be collected at a different sampling date. We placed $350 \mathrm{mg}$ of dried leaf tissue in each compartment and placed two bags per plant genotype randomly in each plot in a $6 \times 6$ grid pattern $(1.25 \mathrm{~m}$ spacing) on 21 September 2012, when O biennis plants were naturally dropping leaves. We used litter from 16 of the 18 original genotypes; there was insufficient material from two genotypes. We collected litter bags at one, two, and six months after placement. For each collection date, we dried the remaining leaf tissue, and weighed the tissue to the nearest $0.1 \mathrm{mg}$. We calculated the decay constant $(k)$ for each bag by taking the negative slope of the linear regression of $\log _{e}$ (fractional mass remaining) vs. time (Schweitzer et al. 2004).

We used a modified method from Wardle et al. (2009) to assess leaf decay in the lab (Appendix B: Fig B1B). This method allowed us to isolate fine-scale differences among plots in their biotic and abiotic soil features from larger scale physical differences in our field leaf decay assay. We filled Petri dishes ( $5.5 \mathrm{~cm}$ diameter) with $8 \mathrm{~g}$ of field soil and added distilled water to reach $40 \%$ moisture content by mass. We then placed $16 \mathrm{mg}$ of dried leaf tissue on a nylon mesh disc on the soil surface, sealed the dish with Parafilm (Bemis, Neenah, Wiscon$\sin$, USA), and placed it in a growth chamber at $25^{\circ} \mathrm{C}$ with a $12 \mathrm{~h}$ photoperiod. We calculated relative mass loss as ([initial mass] - [final mass])/(initial mass). After 60 days, the remaining leaf tissue was gently rinsed with distilled water to remove soil, dried, and weighed to the nearest $0.1 \mu \mathrm{g}$. We chose six plant genotypes to span the range of foliar phenolic content determined in a previous experiment (Johnson et al. 2009a), and crossed leaf litter from each of six genotypes with soil from each of 16 plots in triplicate.

\section{Substrate induced respiration}

We characterized the microbial metabolic potential in soil from each plot, as well as the propensity for $O$. biennis genotypes to affect heterotrophic soil respiration, using substrate induced respiration (SIR) assays (Preston et al. 2012). We chose to measure soil respiration in the lab because we were interested in characterizing the soil metabolic potential using a diverse set of natural and artificial substrates as opposed to in-situ soil respiration. We measured the ambient soil respiration rate from each plot in addition to the respiration rate induced under 20 natural and synthetic substrates (Appendix E: Fig. E1). We selected artificial substrates to span a range of chemical complexity: methyl-cellulose (plant structural component, high molecular weight, complex molecule; Sigma-Aldrich M7140; Sigma-Aldrich, St. Louis, Missouri, USA), gallic acid (a plant phenolic derivative; Sigma-Aldrich G7384), an amino acid mixture (protein derivatives; Sigma-Aldrich R7131), and glucose (cellulose derivative, simple compound with low molecular weight; EMD Chemicals 4074; EMD Chemicals, Gibbstown, New Jersey, USA). Each artificial substrate was adjusted to a standard concentration of $1 \mathrm{mg} \mathrm{C} / \mathrm{mL}$ distilled water and a $\mathrm{pH}$ of 7.0. Our natural substrates were leachates derived from each $O$. biennis genotype, created by steeping $1 \mathrm{~g}$ of dried whole leaf tissue in $1 \mathrm{~L}$ of distilled water for 3 days. We filtered leachates to remove debris using a $0.45-\mu \mathrm{m}$ glass fiber filter. We measured the $\mathrm{pH}$ and total phenolic content of each genotype leachate following the methods of Salminen and Karonen (2011).

We placed $3.5 \mathrm{~g}$ of soil (wet mass from composite field samples) into $30 \mathrm{~mL}$ serum vials, added $1.5 \mathrm{~mL}$ of substrate, capped them with a rubber septum, and sealed them with an aluminum cap (Appendix B: Fig. B1C). Vials were incubated in the dark and gas samples were taken at 0,16 , and $24 \mathrm{~h}$ using a $1-\mathrm{mL}$ syringe after mixing the gas in the vial headspace. We used an infrared gas analyzer (Qubit Systems, Kingston, Ontario, Canada) to determine the $\mathrm{CO}_{2}$ concentration in our samples and calculated the rate of respiration. This sampling procedure was used after a pilot study revealed that the relationship between $\mathrm{CO}_{2}$ production and time was linear and highly consistent over a $48 \mathrm{~h}$ period $\left(R^{2}=0.98, n=36\right.$ samples). We used a completely randomized temporal block design to replicate each soil $(16) \times$ substrate $(21)$ combination three times.

\section{$N$ mineralization rate}

We assessed net nitrogen $(\mathrm{N})$ mineralization rate in the field as a measure of $\mathrm{N}$ cycling that often relates to the amount of $\mathrm{N}$ available to plants (Schimel and 
Bennett 2004). Our methods followed the protocol of Schweitzer et al. (2004). Briefly, on 21 September 2012, we inserted two PVC tubes $(5 \mathrm{~cm}$ radius $\times 15 \mathrm{~cm}$ length, uncovered) in standard locations within each plot to a depth of $10 \mathrm{~cm}$. We removed two soil cores, one as our initial sample and one to incubate in the sunken PVC tube. This process was repeated for each tube. The initial soil sample was frozen at $-20^{\circ} \mathrm{C}$ until 21 November, when we collected the incubated soil samples. We extracted inorganic $\mathrm{N}$ from $5 \mathrm{~g}$ of soil using $50 \mathrm{~mL}$ of $2 \mathrm{~mol} / \mathrm{L} \mathrm{KCl}$. Extracts were shaken on an orbital shaker and filtered (Whatman no. 44). Inorganic $\mathrm{N}$ content was determined using a QuikChem 8500 Flow-Injection Analyzer (Lachat, Loveland, Colorado, USA). Net N mineralization rate was calculated as the difference between final and initial concentrations of $\mathrm{NH}_{4}^{+}-\mathrm{N}$ plus $\mathrm{NO}^{-}-\mathrm{N}$; values were corrected for soil moisture content. Soil $\mathrm{NH}_{4}^{+}-\mathrm{N}$ and $\mathrm{NO}^{-}-\mathrm{N}$ concentration were summed for total inorganic nitrogen production for the incubations. We expressed net $\mathrm{N}$ mineralization rate as a percentage of total soil $\mathrm{N}$ on a per day basis.

\section{Early seedling performance}

To assess potential feedbacks between ecosystem processes and plant performance, we measured early seedling mass of each plant genotype in soil collected from each plot. Recent research shows that seedling performance is a major component of lifetime fitness in $O$. biennis, where genotypic variation in seedling performance alone can predict the evolutionary response of O. biennis populations (Agrawal et al. 2013). In some cases seedling performance is a better predictor of evolution than lifetime seed production (Agrawal et al. 2013; Appendix A: Fig. A1). We used 100-well microcentrifuge tube trays so we could simultaneously manipulate soil and $O$. biennis seedling genotype in a standardized way (Appendix B: Fig. B1D). We filled a tray with soil from a single plot and randomly assigned a seed from one of the 18 genotypes to each cell. In total, we had 16 trays, one for each plot, and we replicated each genotype three times per tray. The trays were placed in a greenhouse $\left(14 \mathrm{~h}\right.$ photoperiod, $26^{\circ} \mathrm{C}$ average daily temperature) and rotated daily to reduce spatial effects. We watered trays by misting from above every 2 $\mathrm{h}$ from 09:00-18:00. Whole seedlings (roots and shoots), were harvested one week after radicle emergence, dried at $55^{\circ} \mathrm{C}$ for $72 \mathrm{~h}$, and weighed to the nearest $0.1 \mu \mathrm{g}$ on a microbalance (XP2U; Mettler Toledo, Mississauga, Ontario, Canada).

\section{Statistical analyses}

Our approach to build models followed the recommendations of Zuur et al. (2009), which involved a stepwise backward elimination procedure to produce a final model. We started with a fully parameterized model and performed a series of likelihood ratio tests (LRTs) on nested models (with and without a single random effect), fit with restricted maximum likelihood (REML) to eliminate random effects with $P>0.10$. We then used a series of LRTs using maximum likelihood to eliminate fixed effects with $P>0.10$. We validated the assumptions of homogeneity of variance and normality by visually examining the distribution of residuals. To test the validity of our statistical approach we used a model selection method based on Akaike information criterion (AIC) scores using the dredge function in the MuMIN package (version 1.12.1) of $\mathrm{R}$. We also used the step function from the package lmerTest (version 2.0-20) to confirm the results of our manual backward selection protocol. Finally we performed parametric bootstrapping with 999 replicates of our fixed and random effect parameters ( $\beta$ coefficients and variance) using the function bootMer from the lme4 package (version 1.1-7).

We used linear mixed-effects (LME) models to analyze our data. The following factors were modeled as fixed effects in our analyses: herbivory treatment (insecticide vs. water control), total $O$. biennis abundance (number of individuals), genotypic composition in each plot (scores along CA 1 and CA 2), and Euclidean distance of each plot. Genotype of the plant litter substrate (hereafter plant genotype), plot (nested within herbivory treatment), and block were modeled as random effects with the exception of the analysis of the lab leaf decay assay, where plant genotype was modeled as a fixed effect because we specifically selected a smaller number of genotypes that varied in phenolic concentration. The effect of plot reflects spatial variation in the biotic and abiotic environment associated with the eight replicate plots within each treatment. We also included terms for each of the two-way interactions involving herbivory treatment, genotypic composition, and plant genotype, and the two-way interaction between $O$. biennis abundance and genotypic composition. We focused on these particular interactions because recent studies have demonstrated that the ecosystem effects of evolution and genetic variation can be contingent on ecological context (Bassar et al. 2010), represented here by the suppression of insect herbivores and the dominance of $O$. biennis within a plot. Interactions involving one random effect were always treated as simple scalar random effects.

We used the lmer function in the lme4 package (version 1.1-7) of R (R Development Core Team 2012) for all analyses. The full statistical model for our ecosystem variables was

$$
\begin{aligned}
& \text { ecosystem variable } \sim H+\mathrm{GC} 1+\mathrm{GC} 2+A+(H \times \mathrm{GC} 1) \\
& +(H \times \mathrm{GC} 2)+(A \times \mathrm{GC} 1)+(A \times \mathrm{GC} 2)+(1 \mid \mathrm{gt}) \\
& +(1 \mid \mathrm{Plot}(H))+(1 \mid H \times \mathrm{gt})+(1 \mid \mathrm{GC} 1 \times \mathrm{gt}) \\
& +(1 \mid \mathrm{GC} 2 \times \mathrm{gt}))
\end{aligned}
$$

where fixed predictor variables include herbivory treatment $(H)$, genotypic composition scores along CA 1 and CA 2 ( $\mathrm{GC} 1$ and $\mathrm{GC} 2$ ), abundance of $O$. biennis $(A)$, interactions between the herbivory treatment and 

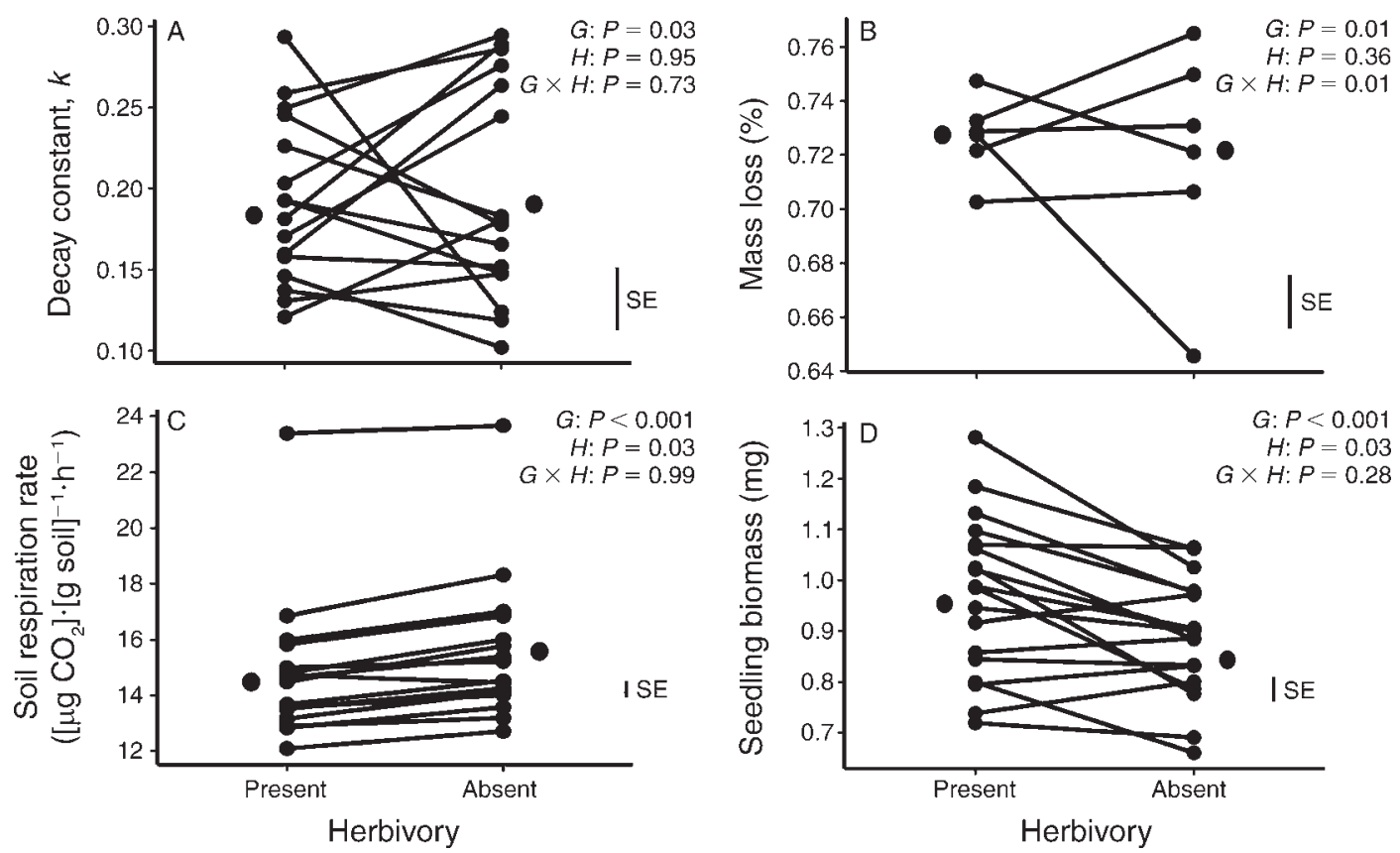

FIG. 2. Reaction norm plots depicting the effects of plant genotype and herbivory on ecosystem responses. (A) Field leaf decay measured as the decay constant $(k)$. (B) Laboratory leaf decay measured as relative loss of litter mass after 60 days. (C) Substrateinduced respiration rate of soils under the addition of leachates derived from the plant genotype litter. (D) Seedling performance quantified as the dry biomass of each genotype seven days post-germination. Each line represents an individual plant genotype, where the ends of the line show genotype means in the presence and absence of herbivory. In each plot, SE represents the average standard error among genotypes, and the circles adjacent to the reaction norms represent the mean values for each herbivory treatment. Significance of the main and interactive effects of plant genotype $(G)$ and herbivory treatment $(H)$ are shown.

genotype composition, and abundance and genotype composition scores. Random predictor variables included plant genotype $(1 \mid \mathrm{gt})$, plot nested within treatment $(1 \mid \operatorname{Plot}(\mathrm{H}))$, and interactions between the herbivory treatment and plant genotype (indicated by $\times$ ), and genotype composition scores and plant genotype. In a separate set of analyses, we included Euclidean distance as a measure of evolution instead of $\mathrm{GCl}$ and $\mathrm{GC} 2$; Euclidean distance was a fixed effect and we included interactions with herbivory treatment, abundance, and plant genotype.

To understand which plant traits might influence ecosystem processes, we used genotype mean trait values measured in a previous experiment to explain variation in ecosystem process rates. The traits measured in the previous experiment include 24 morphological, phenological, and chemical traits (Johnson et al. 2009a).

In general, our different statistical approaches yielded the same results. Specifically, the same factors found to be significant in our backward selection procedure were also retained in our multi-model comparison, and these factors had relatively large model-averaged and bootstrapped parameter estimates. In Appendix C, we present the results from our multi-model comparison (best-fit models, Appendix C: Tables C3 and C4). Next we present and discuss the results from the backwards selection procedure.

\section{Results \\ Leaf decay}

In both the field and the lab, plant genotype of the litter substrate and plot affected leaf decay most strongly (Fig. 2A, B, Appendix C: Table C2). In the field, $O$. biennis genotypes varied twofold $(0.12<k<0.25)$ and plots varied threefold $(0.10<k<0.30)$ in decay rate. In the lab, decay rates varied by $10 \%$ among genotypes and $23 \%$ across plots. By contrast, neither genotypic composition of plots nor suppression of herbivores had independent effects (through impacts on the soil) on the litter decay rates (Fig. 2A, B, Appendix C: Table C2). However, there was a significant herbivory $X$ genotype interaction in the lab assay, indicating that the effect of plant genotype on leaf decay depended on the impact of insect herbivores on soil (Fig. 2B, Appendix C: Table C2). Genotype leaf decay rates were not significantly correlated between the field and lab assays $(r=0.62, P=0.22, N=5)$.

\section{Substrate-induced respiration}

Plant genotype was the most important factor affecting microbial respiration rates, explaining $57 \%$ of the total variation (Fig. 2C, Appendix C: Table C2). We found that insect herbivores caused a small (4\%) decrease in overall soil respiration rates $(P=0.03)$, but did not alter the specific substrate utilization of soil 


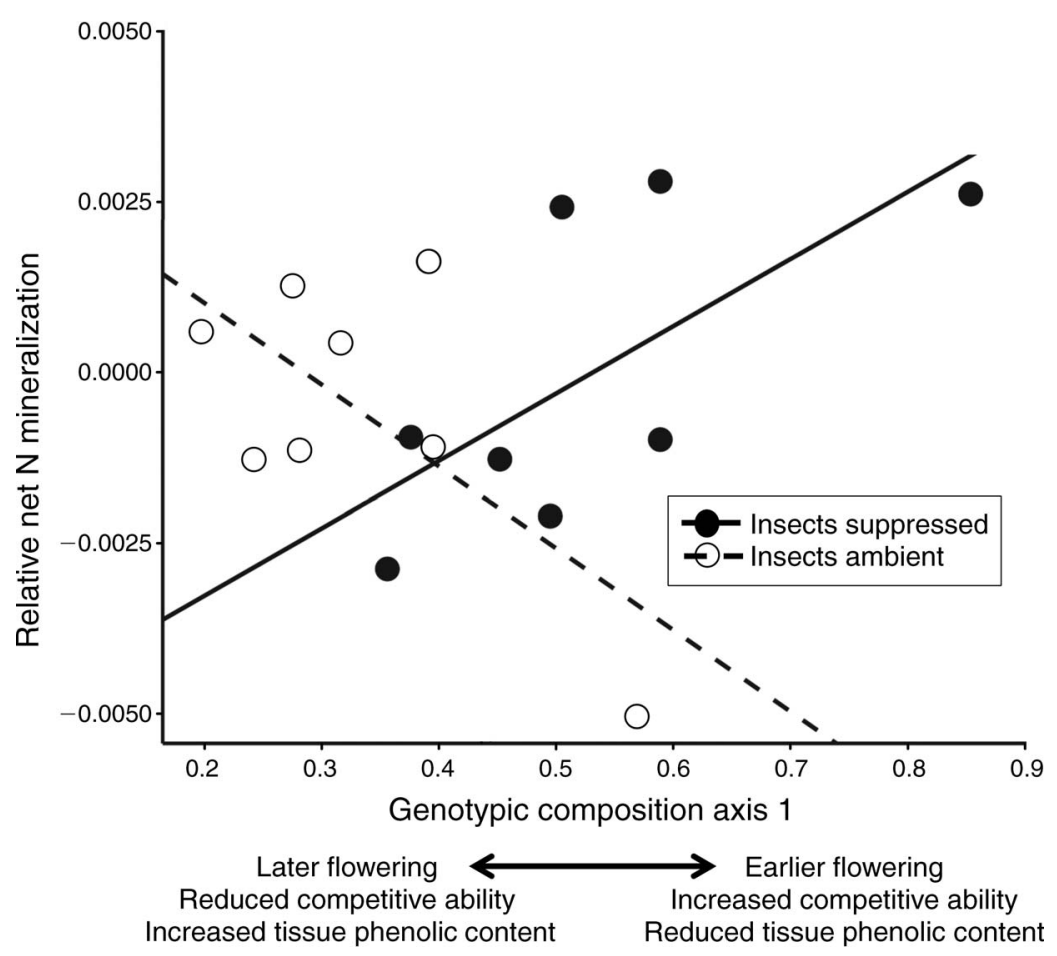

FIG. 3. Soil relative net $\mathrm{N}$ mineralization rate (net $\mathrm{N}$ mineralization rate/total soil $\mathrm{N}$ ) is influenced by an interaction between insect herbivory $(H)$, and genotypic composition $(\mathrm{GC} 1)$. Genotypic composition of $O$. biennis within each plot was quantified as the degree of evolutionary divergence between plots between 2007 and 2011 using correspondence analysis (see Methods). The list of traits below the $\mathrm{GCl}$ axis describes the phenotypic change that occurred across these populations. The relationship between net $\mathrm{N}$ mineralization rate and $O$. biennis plot genotypic composition (axis 1 ) is positive in the absence of herbivores but negative in the presence of herbivores. These results show that herbivory and plant evolution interact to affect soil N cycling. $(H, P=0.61 ; \mathrm{GCl}, P$ $=0.15 ; H \times \mathrm{GC} 1, P<0.001)$.

microbes (nonsignificant herbivory treatment $\times$ substrate interaction), while genotypic composition had no effect (Appendix C: Table C2). Spatial variation across plots had a significant effect on microbial respiration $(P$ $<0.001)$ but explained just $2 \%$ of the total variation (Appendix C: Fig. C1, Table C2).

\section{$N$ mineralization rate}

In contrast to other ecosystem processes, $\mathrm{N}$ mineralization rate was most strongly affected by the presence of herbivores (Fig. 3), and genotypic composition of $O$. biennis populations. The relationship between $\mathrm{N}$ mineralization rate and genotypic composition (axis 1) was reversed under insect herbivore suppression (Fig. 3, Appendix C: Table C1). Even after removing a potential outlier (lowest point from herbivory suppressed plots in Fig. 3), the interaction remained significant $(P=0.03)$.

\section{Early seedling performance}

Early seedling performance was affected by plant genotype and multiple factors through the soil ecosystem, including herbivory treatment, genotypic composition, and plot variation (Fig. 2D, Appendix C: Table C1). We observed nearly twofold variation in seedling mass across genotypes, which explained $16 \%$ of the total variation in seedling performance (Appendix C: Fig. C1). Importantly, this effect was not explained by the average seed mass of genotypes $(P=0.97)$, suggesting that maternal effects are unlikely to explain this effect. Seedlings gained more biomass in soil taken from plots with herbivores (Fig. 2D, Appendix C: Table C1), an effect that explained $4 \%$ of the total variation (Appendix C: Fig. C1). Spatial variation among plots explained a similarly small but significant amount of variation $\left(R^{2}=\right.$ $0.04, P=0.02)$. Finally, $O$. biennis genotypic composition (axis 2) was significantly related to seedling performance (Appendix C: Table C1). Specifically, seedling biomass was negatively related to soil taken from plots with increasing scores along the second axis of genotypic composition. This result shows that the ecosystem-level effects of evolution can feedback to influence seedling performance in the next generation.

\section{Measuring the impact of evolution by Euclidean distance}

We estimated the magnitude or rate of evolutionary change in each plot as the Euclidean distance $(D)$ in genotypic composition between 2007 and 2011 (Appendix B: Table B1). This metric revealed unique effects of evolution on ecosystem processes (Appendix C: Table C2). Plots with greater $D$ had significantly lower rates of 
leaf decay in the field $(P<0.001$, Fig. 4, Appendix C: Table C2). Similarly, leaf decay in the lab assay was reduced on soil taken from plots with greater $D$, but only when $O$. biennis was abundant $(D \times$ abundance $P=$ 0.02 , Appendix C: Table C2). Soil respiration rates were significantly reduced in plots with greater evolution, but only in the presence of insect herbivores $(D \times$ herbivory treatment $P=0.03$, Appendix C: Table C2). Finally, including $D$ as a covariate eliminated the significance of the herbivore treatment on seedling performance (Appendix C: Table C2).

\section{Genetic correlations between plant traits and ecosystem processes}

We detected few significant genetic correlations between plant traits and ecosystem processes. Nevertheless, we found that seedling performance was negatively correlated with total foliar ellagitannin content $(r=$ $-0.50, P=0.03$, Appendix D: Table D1), and a specific flavonoid compound $(r=-0.47, P=0.046$, Appendix D: Table D1), indicating a growth cost to the production of these compounds. Substrate induced soil respiration rates were negatively related to foliar chlorogenic acid content $(r=-0.60, P=0.01$, Appendix D: Table D1, Fig. D1). Litter decay rates were not influenced by any of the plant traits measured. Additionally, we found no correlation between litter decay, substrate induced soil respiration rates, and plant performance, suggesting that the underlying genes responsible for the effects of plant genotype differed for different ecosystem processes.

\section{DiscusSION}

Our primary goals were to understand the relative importance of $O$. biennis plant genotype and contemporary evolution on ecosystem processes, and whether ecosystem consequences of these factors feedback to alter $O$. biennis seedling performance in future generations. Plant genotype strongly affected multiple ecosystem processes, whereas the ecosystem effects of contemporary evolution depended on our metric of evolution (i.e., genotypic composition or Euclidean distance) and frequently interacted with other factors (e.g., presence of herbivores and focal plant density). We also detected direct effects of contemporary evolution on leaf decay in the field and seedling performance in the next generation. The latter result suggests that ecosystem-evolutionary feedbacks exist between $O$. biennis populations and their soil environments. The genotypic and evolutionary effects we observed were of similar magnitude to the ecosystem effects of insect herbivory and plot variation. Below we discuss the importance of these results in the context of (1) the ecosystem-level consequences of genetic variation and evolution, (2) the relative importance of ecological and evolutionary factors in shaping ecosystem processes, and (3) feedbacks between ecosystem processes and plant performance.

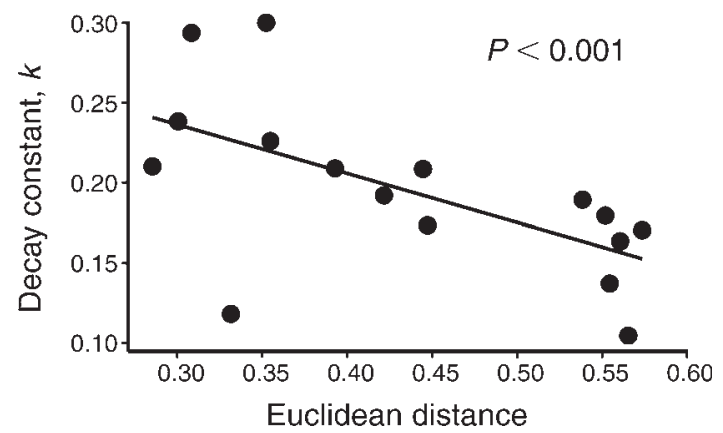

FIG. 4. The magnitude of evolutionary change occurring in $O$. biennis populations negatively affects leaf decay rate in the field $\left(R^{2}=0.30, P<0.001\right)$. The magnitude of evolutionary change was calculated as the Euclidean distance in genotypic composition from 2007 to 2011. Euclidean distance measures the magnitude or rate of evolutionary change within a population irrespective of direction. Each point represents the leaf decay rate ( $k$; see Methods) averaged across $16 \mathrm{O}$. biennis genotypes replicated in litter bags in each plot.

\section{Extended effects of plant genotype and evolution on ecosystems}

Recent research shows that intraspecific genetic variation can influence litter decay and nutrient cycling (Schweitzer et al. 2004, Madritch et al. 2006). We corroborate these findings by showing that differences among $O$. biennis genotypes affected the rate of leaf decay (Fig. 1B, C). We also present the novel result that genotypic differences in litter leachates, an important source of carbon and nutrients in terrestrial ecosystems (Gosz et al. 1973), strongly affect microbial activity measured as soil respiration (Fig. 1D). Previous work showed that total litter $\mathrm{C}$ lost by soluble leaching varied between 6\% and 39\% among plant species (Magill and Aber 2000). Our results imply that heritable variation within species can influence total litter carbon and nutrient cycling as much as variation among species.

We investigated the ecosystem effects of evolution using two metrics of evolutionary change. Genotypic composition quantified using correspondence analysis measured the direction of evolutionary change across our populations of $O$. biennis and affected $\mathrm{N}$ cycling (Fig. 3). Divergent populations along genotypic composition axis 1 differed in average flowering date, competitive ability, and tissue phenolic content (Agrawal et al. 2012). These phenotypic differences could affect the timing of $\mathrm{N}$ uptake, $\mathrm{N}$ use efficiency and acquisition strategies, and substrate quality, including the amount of labile $\mathrm{C}$ and $\mathrm{N}$ across populations, all of which could influence the net release or immobilization of labile $\mathrm{N}$ in soil (Chapman et al. 2006). Euclidean distance quantified the magnitude or rate of evolutionary change, irrespective of direction, and it was associated with reduced leaf decay and soil respiration rates (Fig. 4). If evolutionary change within a focal plant population leads to a mismatch between substrates and decomposer communities, then populations undergoing 
greater evolutionary change may disrupt ecosystem processes (Ayres et al. 2009). In our case, this negative effect of the rate of evolution on leaf decay and soil respiration may be transient, as the associated communities of decomposer organisms become better adapted to the substrates supplied by the focal population undergoing evolution.

Our results show that the ecosystem effects of evolution are dependent on the way evolution is measured. In our case, the ecosystem effects of the direction ( $\mathrm{GC} 1$ and $\mathrm{GC} 2$ ) and magnitude (Euclidean distance) of evolutionary change were complementary yet not equivalent, as they revealed different effects on ecosystems (Appendix C: Table C1, C2). Our results also indicate the ecosystem effects of evolution are contingent on ecological context. The effects of plant evolutionary change on $\mathrm{N}$ mineralization and soil respiration rate were modified by the presence of insect herbivores. Moreover, while evolution had a direct effect on leaf decay in the field, its effect in the lab was significantly stronger in treatments with soil taken from plots with high $O$. biennis abundance.

\section{The relative importance of multiple factors affecting ecosystem processes}

Our results indicate that plant genotype can be an important factor influencing terrestrial ecosystems (Fig. 1, Appendix C: Fig. C1). Specifically, we show that plant genotype strongly affects leaf decay and soil respiration, potentially altering the soil communities mediating these processes over time. In two previous studies, the effects of plant genotype on leaf decay were weaker than effects of variation among plant species and nutrient deposition (Madritch et al. 2006, Crutsinger et al. 2009). However, others have found that the effect of intraspecific variation on leaf decay was comparable to the effects of among-plot spatial variation (Madritch and Hunter 2002), and herbivore-induced changes in leaf tissues (Schweitzer et al. 2005).

Compared to insecticide-treated plots, the presence of herbivores played a role in $\mathrm{N}$ cycling, soil respiration, yet had no effect on leaf decay (Appendix C: Table C1). We found that the effect of insect herbivores on $\mathrm{N}$ cycling is complex, involving an interaction with $O$. biennis genotypic composition (Fig. 3). The presence of insect herbivores could directly or indirectly alter the amount of labile $\mathrm{C}$ and $\mathrm{N}$ entering the soil environment (Classen et al. 2013) and the composition of decomposer communities (Classen et al. 2006). Insect herbivores, in addition to the genotypic composition of the resident plant population, could contribute in a nonadditive fashion to produce the interactive effect of insect herbivores and genotypic composition on $\mathrm{N}$ cycling. In a similar fashion the presence of insect herbivores may interact with the rate of evolution to alter soil respiration rates (Appendix $\mathrm{C}$ : Table $\mathrm{C} 2$ ). Our results demonstrate that multiple evolutionary and ecological factors can directly and indirectly influence multiple ecosystem processes via complex interactions.

\section{Feedbacks to plant fitness}

Ecosystem-evolutionary feedbacks have been hypothesized to occur in nature (Fussmann et al. 2007), but empirical evidence for them has been restricted to microbial microcosm studies (Lennon and Martiny 2008, Gravel et al. 2012, Lawrence et al. 2012). Evolution by natural selection in plant populations can alter ecosystem properties that then feedback to affect focal plant fitness in future generations if two conditions are satisfied. First, traits subject to natural selection must induce changes in the abiotic (Bassar et al. 2010) or biotic environment (Johnson et al. 2009b). Second, these changes in the environment must influence the fitness of focal individuals in subsequent generations (Kylafis and Loreau 2008). Our findings are consistent with this scenario and could be the result of variation in either the biotic (e.g., soil community) or abiotic (e.g., N availability) soil environment caused by evolution in O. biennis populations. We acknowledge that seedling performance is just one component of plant performance, but in $O$. biennis it represents a crucial life stage where seedling performance predicts evolutionary responses. Our results build on past studies showing that differences among soil communities harbored by closely related plant individuals can have consequences for plant performance and nutrient cycling (Schweitzer et al. 2004, Pregitzer et al. 2010). The prevalence and importance of ecosystem-evolutionary feedbacks remains poorly characterized and represents an important frontier for future research.

\section{Caveats}

Although our results show clear effects of plant genotype and evolution on ecosystem processes, there are several limitations of our study that help define paths of future research. First, O. biennis is only one of many plant species occurring in our field plots. Other plant species responded to herbivore suppression and it is unclear how they affected ecosystems. For example, Agrawal et al. (2012) demonstrated that dandelion (Taraxaxum officinale) increased in abundance under herbivore suppression and caused $O$. biennis populations to evolve increased competitive ability. Although not apparent from our plant community survey (Appendix B: Table B2), subtle shifts in the relative abundance or even evolutionary change of co-occurring species could directly and indirectly impact the ecosystem processes we measured. This highlights the importance of moving beyond the single-species framework used here to the reality of natural communities where the dynamics within and between coexisting and potentially codominant species are driving ecosystem processes (Whitlock et al. 2011). The reciprocal influences between genetic diversity and species diversity highlight the potential role of interactions between evolutionary 
change and community dynamics (Vellend and Geber 2005).

Second, our characterization represents a single snapshot of the ecosystem effects of evolution. These effects may diminish or strengthen over time, which may depend on the abundance of the focal populations undergoing evolution and the contribution of extinction/ colonization events of other community members to ecosystem processes. In our plots the abundance of $O$. biennis declined over time and thus the strength of its ecosystem effects is likely declining too. Nonetheless, there may be legacy effects of the evolutionary change in populations of dominant species, even if their populations are ephemeral, a question that remains largely unanswered. Finally, a large proportion of the variation in most ecosystem processes remained unexplained, which could be due to the factors discussed previously.

\section{Conclusions}

We have shown that genetic variation in a single plant species has comparable effects on ecosystem processes as biotic and abiotic ecological factors that are more commonly identified as drivers of ecosystems. We also provide evidence that contemporary evolution can have ecosystem-level effects in the field and often these effects interact with other factors to influence ecosystem functioning. Thus, the importance of evolution within plant populations for ecosystem processes is not straightforward and likely depends on the ecological dominance of the focal population, the traits undergoing evolution, and the ecological context. Our demonstration that the extended effects of plant evolution on soil properties can feedback to alter plant performance in future generations suggests that reciprocal effects of evolution and ecosystem processes may be pervasive and self-perpetuating.

\section{ACKNOWLEDGMENTS}

We thank John Maron and Juha-Pekka Salminen for their contributions to the long-term herbivore exclusion experiment. We thank A. De Serrano, S. Gagliardi, R. Godfrey, M. Kalich, A. Lochab, G. Noyce, B. Pitton, and C. Thomsen for support in the field, laboratory and greenhouse. The paper was improved by comments from D. Anstett, R. Godfrey, M. Turcotte, N. Turley, and especially P. Adler, and two anonymous reviewers. This work was funded by a National Science Foundation grant (EAGER - 0950231) to A. Agrawal and M. Johnson, as well as an NSERC Discovery grant, a Canadian Foundation for Innovation LOF grant and Ontario Research Fund grant to M. Johnson.

\section{Literature Cited}

Agrawal, A. A., A. P. Hastings, M. T. J. Johnson, J. L. Maron, and J. P. Salminen. 2012. Insect herbivores drive real-time ecological and evolutionary change in plant populations. Science 338:113-116.

Agrawal, A. A., M. T. J. Johnson, A. P. Hastings, and J. L. Maron. 2013. A field experiment demonstrating plant lifehistory evolution and its eco-evolutionary feedback to seed predator populations. American Naturalist 181:S35-S45.

Ayres, E., H. Steltzer, B. L. Simmons, R. T. Simpson, J. M. Steinweg, M. D. Wallenstein, N. Mellor, W. J. Parton, J. C.
Moore, and D. H. Wall. 2009. Home-field advantage accelerates leaf litter decomposition in forests. Soil Biology and Biochemistry 41:606-610.

Bailey, J. K., et al. 2009. From genes to ecosystems: a synthesis of the effects of plant genetic factors across levels of organization. Philosophical Transactions of the Royal Society B 364:1607-1616.

Bardgett, R. D., and D. A. Wardle. 2010. Abovegroundbelowground linakges: biotic interactions, ecosystem processes, and global change. Oxford University Press, Oxford, UK.

Bassar, R. D., et al. 2010. Local adaptation in Trinidadian guppies alters ecosystem processes. Proceedings of the National Academy of Sciences USA 107:3616-3621.

Chapman, S. K., J. A. Langley, S. C. Hart, and G. W. Koch. 2006. Plants actively control nitrogen cycling: uncorking the microbial bottleneck. New Phytologist 169:27-34.

Classen, A. T., S. K. Chapman, T. G. Whitham, S. C. Hart, and G. W. Koch. 2013. Long-term insect herbivory slows soil development in an arid ecosystem. Ecosphere 5:1-14.

Classen, A. T., J. Demarco, S. C. Hart, T. G. Whitham, N. S. Cobb, and G. W. Koch. 2006. Impacts of herbivorous insects on decomposer communities during the early stages of primary succession in a semi-arid woodland. Soil Biology and Biochemistry 38:972-982.

Cleland, R. 1972. Oenothera: cytogenetics and evolution. Academic Press, New York, New York, USA.

Crutsinger, G. M., B. E. Carter, and J. A. Rudgers. 2013. Soil nutrients trump intraspecific effects on understory plant communities. Oecologia 173:1531-1538.

Crutsinger, G. M., M. D. Collins, J. A. Fordyce, Z. Gompert, C. C. Nice, and N. J. Sanders. 2006. Plant genotypic diversity predicts community structure and governs an ecosystem process. Science 313:966-968.

Crutsinger, G. M., N. J. Sanders, and A. T. Classen. 2009. Comparing intra- and inter-specific effects on litter decomposition in an old-field ecosystem. Basic and Applied Ecology 10:535-543.

Fierer, N., J. P. Schimel, R. G. Cates, and J. Zou. 2001. Influence of balsam poplar tannin fractions on carbon and nitrogen dynamics in Alaskan taiga floodplain soils. Soil Biology and Biochemistry 33:1827-1839.

Fussmann, G. F., M. Loreau, and P. A. Abrams. 2007. Ecoevolutionary dynamics of communities and ecosystems. Functional Ecology 21:465-477.

Geber, M. A., and L. R. Griffen. 2003. Inheritance and natural selection on functional traits. International Journal of Plant Sciences 164:S21-S42.

Gosz, J. R., G. E. Likens, and F. H. Bormann. 1973. Nutrient release from decomposing leaf and branch litter in the Hubbard Brook Forest, New Hampshire. Ecological Monographs 43:173-191.

Gravel, D., T. Bell, C. Barbera, T. Bouvier, T. Pommier, P. Venail, and N. Mouquet. 2012. Experimental niche evolution alters the strength of the diversity-productivity relationship. Nature 469:89-92.

Harmon, L. J., B. Matthews, S. Des Roches, J. M. Chase, J. B. Shurin, and D. Schluter. 2009. Evolutionary diversification in stickleback affects ecosystem functioning. Nature 458:11671170.

Hersch-Green, E. I., N. E. Turley, and M. T. J. Johnson. 2011. Community genetics: what have we accomplished and where should we be going? Philosophical Transactions of the Royal Society B 366:1453-1460.

Hunter, M. D. 2001. Insect population dynamics meets ecosystem ecology: effects of herbivory on soil nutrient dynamics. Agricultural and Forest Entomology 3:77-84.

Johnson, M. 2011. The contribution of evening primrose (Oenothera biennis) to a modern synthesis of evolutionary ecology. Population Ecology 53:9-21. 
Johnson, M. T. J., A. A. Agrawal, J. L. Maron, and J. P. Salminen. 2009a. Heritability, covariation and natural selection on 24 traits of common evening primrose (Oenothera biennis) from a field experiment. Journal of Evolutionary Biology 22:1295-1307.

Johnson, M. T. J., M. Vellend, and J. R. Stinchcombe. $2009 b$. Evolution in plant populations as a driver of ecological changes in arthropod communities. Philosophical Transactions of the Royal Society B 364:1593-1605.

Kylafis, G., and M. Loreau. 2008. Ecological and evolutionary consequences of niche construction for its agent. Ecology Letters 11:1072-1081.

Lawrence, D., F. Fiegna, V. Behrends, J. G. Bundy, A. B. Phillimore, T. Bell, and T. G. Barraclough. 2012. Species interactions alter evolutionary responses to a novel environment. PLoS Biology 10(5):e1001330.

Lennon, J. T., and J. B. H. Martiny. 2008. Rapid evolution buffers ecosystem impacts of viruses in a microbial food web. Ecology Letters 11:1178-1188.

Madritch, M., J. R. Donaldson, and R. L. Lindroth. 2006. Genetic identity of Populus tremuloides litter influences decomposition and nutrient release in a mixed forest stand. Ecosystems 9:528-537.

Madritch, M. D., and M. D. Hunter. 2002. Phenotypic diversity influences ecosystem functioning in an oak sandhills community. Ecology 83:2084-2090.

Magill, A. H., and J. D. Aber. 2000. Dissolved organic carbon and nitrogen relationships in forest litter as affected by nitrogen deposition. Soil Biology and Biochemistry 32:606613.

Matthews, B., et al. 2011. Toward an integration of evolutionary biology and ecosystem science. Ecology Letters 14:690701.

Post, D. M., and E. P. Palkovacs. 2009. Eco-evolutionary feedbacks in community and ecosystem ecology: interactions between the ecological theatre and the evolutionary play. Philosophical Transactions of the Royal Society B 364:16291640.

Pregitzer, C. C., J. K. Bailey, S. C. Hart, and J. A. Schweitzer. 2010. Soils as agents of selection: feedbacks between plants and soils alter seedling survival and performance. Evolutionary Ecology 24:1045-1059.

Preston, M. D., K. A. Smemo, J. W. McLaughlin, and N. Basiliko. 2012. Peatland microbial communities and decomposition processes in the James Bay lowlands, Canada. Frontiers in Microbiology 3:1-15.
R Development Core Team. 2012. R version 3.1.2. R Project for Statistical Computing, Vienna, Austria. www. r-project.org

Salminen, J. P., and M. Karonen. 2011. Chemical ecology of tannins and other phenolics: we need a change in approach. Functional Ecology 25:325-338.

Schimel, J. P., and J. Bennett. 2004. Nitrogen mineralization: challenges of a changing paradigm. Ecology 85:591-602.

Schlesinger, W. H., J. A. Raikes, A. E. Hartley, and A. F. Cross. 1996. On the spatial pattern of soil nutrients in desert ecosystems. Ecology 77:364-374.

Schweitzer, J. A., J. K. Bailey, S. C. Hart, and G. M. Wimp. 2005. The interaction of plant genotype and herbivory decelerate leaf litter decomposition and alter nutrient dynamics. Oikos 110:133-145.

Schweitzer, J. A., J. K. Bailey, B. J. Rehill, G. D. Martinsen, S. C. Hart, R. L. Lindroth, P. Keim, and T. G. Whitham. 2004. Genetically based trait in a dominant tree affects ecosystem processes. Ecology Letters 7:127-134.

Tack, A. J. M., M. T. J. Johnson, and T. Roslin. 2011. Sizing up community genetics: it's a matter of scale. Oikos 121:481488.

Vaieretti, M. V., A. M. Cingolani, and N. P. Harguindeguy. 2013. Effects of differential grazing on decomposition rate and nitrogen availability in a productive mountain grassland. Plant and Soil 371:675-691.

Vellend, M., and M. A. Geber. 2005. Connections between species diversity and genetic diversity. Ecology Letters 8:767781.

Wardle, D. A., R. D. Bardgett, L. R. Walker, and K. I. Bonner. 2009. Among- and within-species variation in plant litter decomposition in contrasting long-term chronosequences. Functional Ecology 23:442-453.

Whitham, T. G., et al. 2006. A framework for community and ecosystem genetics: from genes to ecosystems. Nature Reviews Genetics 7:510-523.

Whitlock, R., M. C. Bilton, J. P. Grime, and T. Burke. 2011. Fine-scale community and genetic structure are tightly linked in species-rich grasslands. Philosophical Transactions of the Royal Society B 366:1346-1357.

Yang, L. H., and C. Gratton. 2014. Insects as drivers of ecosystem processes. Current Opinion in Insect Science 2:2632.

Zuur, A. E. N. Ieno, N. Walker, A. A. Saveliev, and G. M. Smith. 2009. Mixed effects models and extensions in ecology with R. Springer, New York, New York, USA.

\section{SupPlemental Material}

\section{Ecological Archives}

Appendices A-E are available online: http://dx.doi.org/10.1890/14-2333.1.sm 